\title{
Thermomechanical properties of the silanized-kenaf/ polystyrene composites
}

\author{
Y. Xu, S. Kawata, K. Hosoi, T. Kawai, S. Kuroda* \\ Department of Production Science and Technology, Graduate School of Engineering, Gunma University, 29-1 hon-cyo, \\ Ota, Gunma 373-0057, Japan
}

Received 12 June 2009; accepted in revised form 11 August 2009

\begin{abstract}
In order to improve the poor interfacial adhesion of the kenaf fiber and polystyrene (PS) in their composite material, the surface of the kenaf fiber was modified using a synthesized polymeric coupling agent to promote adhesion with PS matrix. The dynamic thermo-mechanical properties of the composite composed of modified kenaf fiber and PS were also investigated. The polymeric coupling agent treatment of the kenaf fiber increased the fiber-matrix interaction through a condensation reaction between alkoxysilane and hydroxyl groups of kenaf cellulose. DMA (Dynamic Mechanical Thermal Analysis) results showed that the modified fiber composites have higher $E^{\prime}$ and lower tan $\delta$ than those with untreated fiber indicating that a greater interfacial interaction between the matrix resin and the fiber. It was also found that the storage modulus increases in proportion with the $\mathrm{Si} / \mathrm{C}$ ratio on the fiber surface.
\end{abstract}

Keywords: polymer composites, kenaffiber, polymeric coupling agent, dynamic mechanical properties, interfacial interaction

\section{Introduction}

Biodegradable or environmentally acceptable materials have attained increasing interest over the past decades due to environmental pressure derived from the consumption of the petroleum-based materials [1-3]. The use of natural fibers, derived from annually renewable resources, as reinforcing fibers in both thermoplastic and thermoset matrix composites provides positive environmental benefits with respect to ultimate disposability and raw material utilization. Other benefits are also seen as the natural fibers absorb nitrogen and phosphorus included in the soil [4-7].

Kenaf, Hibiscus cannabinus, L. family Malvaceae, is an herbaceous annual plant that can be grown under a wide range of weather condition, especially in Asia and Central America and finds application as a cordage and sack fiber. The excellent cellulose in the kenaf fiber (KF) source also made the kenaf as a source for a large range of paper products. Recently, researches have further increased the diversity of uses for kenaf by demonstrating its suitability in building materials, textiles, adsorbents, and fibers in new and recycled plastics [8]. KF shows low density, high impact resistance, and high modulus. In addition, KF is available in large amounts, easy to get, low cost, non-abrasive during processing, it exhibits high specific mechanical properties and biodegradability [9-13]. However the hydrophilicity of natural fibers results in high moisture absorption and weak adhesion to hydrophobic matrix resin. And there also have been other challenges presented by natural fibers such as large variability of mechanical properties, low ultimate strength, lower elongation, problems with nozzle flow in injection molding machines, bubbles

*Corresponding author, e-mail: skuroda@gunma-u.ac.jp

(C) BME-PT 
in the product, and poor resistance to weathering [14-18]. To increase the interaction between the fibers and the resin is the way to tackle with the low adhesion. Normally, there are two ways to treat KF to make it compatible with resin matrix: one is alkaline treatment, by using an alkaline solution, which can dissolve unwanted microscopic pits on the fibers resulting in better fiber matrix adhesion. The other way is using coupling agent. The coupling agent has two functions: to react with $-\mathrm{OH}$ groups of the cellulose and to react with the functional groups of the matrix with the goal of facilitating stress transfer between the fibers and the matrix. The commonly used coupling agent includes maleic andydride, silane coupling agent, etc [19$22]$. The effect of the coupling agent was only concluded form the phenomenon of the improvement of the mechanical properties of the composite. Little research is available for the quantitative effect of the coupling agent on the mechanical properties [23-25]. Recently we have synthesized a novel coupling agent composed of methacryloxypropyltriethoxysilane by means of photo-graft polymerization. In this paper, the effect of the coupling agent is studied through quantitative analysis of the reactivity of KF with coupling agent.

\section{Experiments}

\subsection{Material}

Kenaf fiber (KF) used in this experiment was harvested in Henan Province, China. The fiber was cut into $2-5 \mathrm{~mm}$ length, and was washed by running water and then dried up in oven at $80^{\circ} \mathrm{C}$ until constant weight.

A commercial-grade polystyrene $(\mathrm{PSJ} 433, \mathrm{MFI}=$ $21.0 \mathrm{~g} / 10 \mathrm{~min}$ at $230^{\circ} \mathrm{C} / 2.16 \mathrm{~kg}, E^{\prime}=0.8 \mathrm{GPa}, \rho=$ $1.0304 \mathrm{~g} / \mathrm{cm}^{3}, v=0.33$, PS Japan) was selected as polymer matrix.

The polymeric coupling agent (CA) was synthesized in our lab. Figure 1 shows the structure of $\mathrm{CA}$ used in this study.

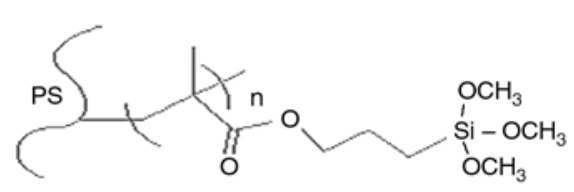

Figure 1. Structure of the coupling agent

\subsection{Production of Coupling Agent (CA)}

PS powder was supplied from Toyo Styrene Co, Ltd. The powder size is around $200 \mu \mathrm{m}$.

3-methacryloxypropyltriethoxysilane (MAPTMS) from Shin-Etsu Silicones was used as grafting monomer and used as received.

Xanthone was used as photo-initiator. Toluene and methanol were used as solvent.

Xanthone was dissolved in methanol/toluene mixture solvent to prepare initiator solution. The solvent and monomer were put into the reactor to form a solution, and then PS powder was put into the reactor. The reactor was bubbled with nitrogen for $1 \mathrm{~h}$.

The reactors containing monomer, initiator, polymer matrix and solvent was then heated to $60^{\circ} \mathrm{C}$, and irradiated with a $400 \mathrm{~W}$ high pressure $\mathrm{Hg}$ lamp (Riko, UVL-400H, Riko Kagaku, Japan) for $4 \mathrm{~h}$.

After the photo-graft polymerization, the PS powder was washed with methanol in a Soxhlet extractor for $24 \mathrm{~h}$ and then dried at room temperature in a vacuum oven for $48 \mathrm{~h}$.

The grafting of MAPTMS onto PS powder was confirmed by the FT-IR spectra recorded on JASCO FT/IR-8000 and the grafting yield (GY) was evaluated by Equation (1):

Grafting yield $[\%]=\left(\frac{W_{1}-W_{0}}{W_{0}}\right) 100$

where, $W_{1}$ is the weight PS powder after grafting and $W_{0}$ is the weight of the original weight of PS powder.

\subsection{Reaction of CA with KF in THF solvent}

First, KF was washed in THF solvent at $70^{\circ} \mathrm{C}$ for $1 \mathrm{~h}$ to remove impurities. And then the CA was dissolved in THF at different concentration to react with $\mathrm{KF}$ at $70^{\circ} \mathrm{C}$ for $1 \mathrm{~h}$. The modified $\mathrm{KF}$ was filtered and then dried in a vacuum oven until constant weight.

\subsection{Preparation of composites using a kneader}

PS/KF/CA composites were prepared using a kneader (Labo Plastomill 4C, Toyoseiki, Japan). The PS and the KF reacted with CA were weighted as 50 to 50 . To prepare the composites, the mixing 
Table 1 Composition of the samples

\begin{tabular}{|l|c|l|}
\hline Sample code & $\begin{array}{c}\text { PS } \\
\text { [weight ratio] }\end{array}$ & \multicolumn{1}{|c|}{$\begin{array}{c}\text { KF } \\
\text { [weight ratio] }\end{array}$} \\
\hline PSJ433 & 100 & 0 \\
\hline C0 & 50 & 50 (THF-washed) \\
\hline C1 & 50 & 50 (reacted with 5 wt\% CA) \\
\hline C2 & 50 & 50 (reacted with $10 \mathrm{wt} \%$ CA) \\
\hline C3 & 50 & 50 (reacted with $15 \mathrm{wt} \%$ CA) \\
\hline
\end{tabular}

temperature was set at $195^{\circ} \mathrm{C}$. PS was first added into the kneader at $22 \mathrm{rpm}$ and after the PS was melted, the reacted KF was mixed with the molten PS for $10 \mathrm{~min}$ at $30 \mathrm{rpm}$. After blending, the compound was hot-pressed at $30 \mathrm{MPa}$ for $5 \mathrm{~min}$, and then moved to cooling press until the temperature of the compound was cooled down to room temperature under a pressure of $25 \mathrm{MPa}$. The composition of the samples prepared is listed in Table 1 with their code.

\subsection{Recovery of KF from composites}

The PS/KF/CA composites were dissolved in THF solvent for $5 \mathrm{~h}$ at room temperature, and then the solution was filtered to recover the kneaded KF fiber. The recovered KF was then washed with THF at least twice to clean the fiber surface, and dried in a vacuum oven until constant weight. The diameter, the length and the surface composition of the KF fiber before and after kneading were analyzed by digital microscope, scanning electron microscope (SEM) and energy dispersive X-ray spectrometer (SEM-EDX).

\subsection{Measurements}

The FTIR spectra of the coupling agent was recorded on Fourier-transform IR spectrophotometer (JASCO FT/IR - 8000). Samples were mixed with analytical grade $\mathrm{KBr}$ and compressed into disk and the spectra were recorded with a $2 \mathrm{~cm}^{-1}$ resolution and 32 scans.

The fiber diameter before and after kneading was detected by digital microscope VHX-600 (Keyence, Japan). A maximum of 200 magnifications were tested for each sample.

The density of the KF was detected by a density analytical balance AUW120D (Shimadzu corporation, Japan) with using 2-propanol for the liquid medium.
Samples were studied by dynamic mechanical analysis (DMA) using tensile mode with Rheolograph Solid S-1 (Toyoseiki, Japan). The measurements were carried out at temperature range from 30 to $150^{\circ} \mathrm{C}$ at a heating rate of $2^{\circ} \mathrm{C} / \mathrm{min}$. The test frequency was $10 \mathrm{~Hz}$ and the sample size was $20 \mathrm{~mm} \times 5 \mathrm{~mm} \times 1.5 \mathrm{~mm}$. The storage modulus, loss modulus and $\tan \delta$ of the samples were recorded during the running.

Scanning electron microscope and energy dispersive X-ray spectrometer (SEM-EDX) $(\mathrm{S}-3000 \mathrm{~N}$, Hitachi; EX-200K Horiba) was used to measure the element of the surface on the KF, the recovered KF and the KF reacted with CA in THF solvent. The measurements were carried out at $15 \mathrm{kV}$ and the analysis depth is about $1 \mu \mathrm{m}$.

\section{Results and discussion}

\subsection{Characterization of CA}

Figure 2 is a typical FTIR spectrum of CA. The strong peak appeared at 1089, 1602 and $1729 \mathrm{~cm}^{-1}$ which correspond to the absorption of $\mathrm{Si}-\mathrm{O}-\mathrm{C}$, benzene ring and carbonyl group, respectively. The grafting yield was calculated using Equation (1) and the estimated GY for the present CA was 5.8\%, which was found to be consistent with the values estimated by Nuclear Magnetic Resonance (NMR) and FT-IR measurements as reported elsewhere [26].

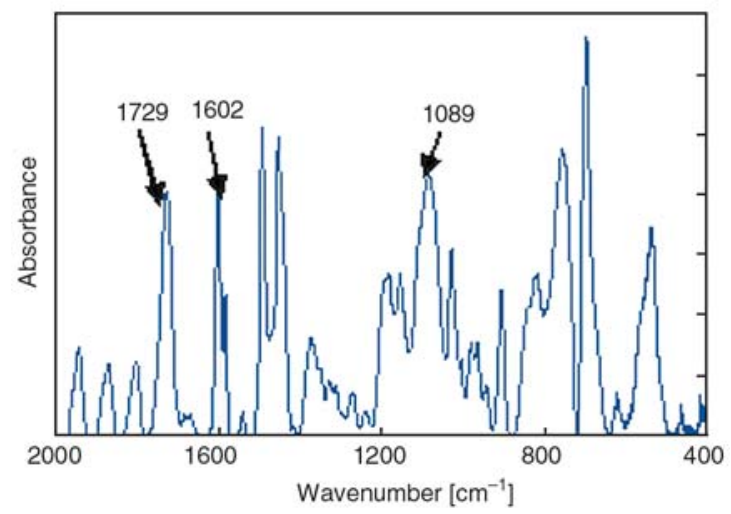

Figure 2. Typical FT-IR spectrum of the coupling agent

\subsection{Surface elements analysis of the kenaf fiber}

The surface of the KF was modified using the synthesized polymeric coupling agent in THF solution to promote the adhesion with the PS matrix. The 


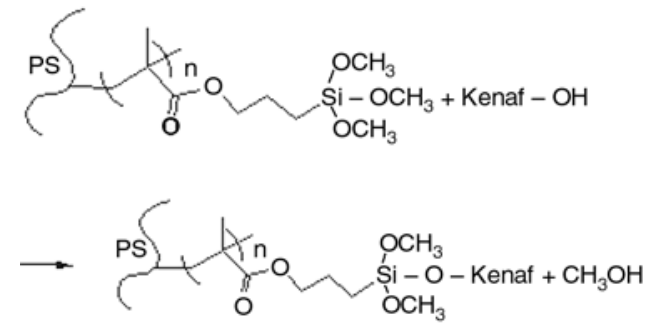

Figure 3. Possible reaction mechanism of the coupling agent with kenaf fiber

main component of $\mathrm{KF}$ is cellulose of which hydroxyl group is to be reacted with CA in the following mechanism shown in Figure 3.

The reaction of coupling agent with KF is the esterexchange reaction between the alkoxysilane groups (Si-OR) and hydroxyl groups of KF, which results in the KF surface covered with the hydrophobic PS chain of the CA that can entangle with PS matrix to enhance the interfacial adhesion between KF and PS matrix.

The KF fibers before and after kneading were shown in Figure 4. The KF fiber diameter before kneading is around $50-120 \mu \mathrm{m}$. After kneading, the diameter of the KF fiber was kept around 50$100 \mu \mathrm{m}$. It shows that the diameter of the KF fiber did not change during kneading but the fiber length decreased down to c.a. $400-500 \mu \mathrm{m}$.

$\mathrm{Si} / \mathrm{C}$ ratio of the KF recovered from the composites and the KF reacted with CA are shown in Figure 5. It can be seen along with the increasing of the $\mathrm{CA}$ weight fraction, the $\mathrm{Si} / \mathrm{C}$ ratio of the reacted $\mathrm{KF}$ is increased obviously. After the CA weight fraction reached $10 \mathrm{wt} \%$, the $\mathrm{Si} / \mathrm{C}$ ratio achieves saturation and it is not increased any more.

The $\mathrm{Si} / \mathrm{C}$ ratio of the recovered $\mathrm{KF}$ is lower than the modified KF fiber, which indicates that the silicon combined with $\mathrm{KF}$ has been removed during kneading. It should be noted that the recovered KF had reduced length of c.a. $400-500 \mu \mathrm{m}$ while the diameter did not change indicating some breakage occurred during the process.

However, the KF reacted in $15 \%$ CA solution seems to form more chemical bonds with a CA molecular chain than that reacted in $10 \%$ solution even though the number of CA molecule attached to a $\mathrm{KF}$ are similar to each other so that the CA molecule remains on $\mathrm{KF}$ when the neighbor fiber surface is peeled.

CA has a PS main chain, which could entangle with a matrix PS molecular chain as well as set up van

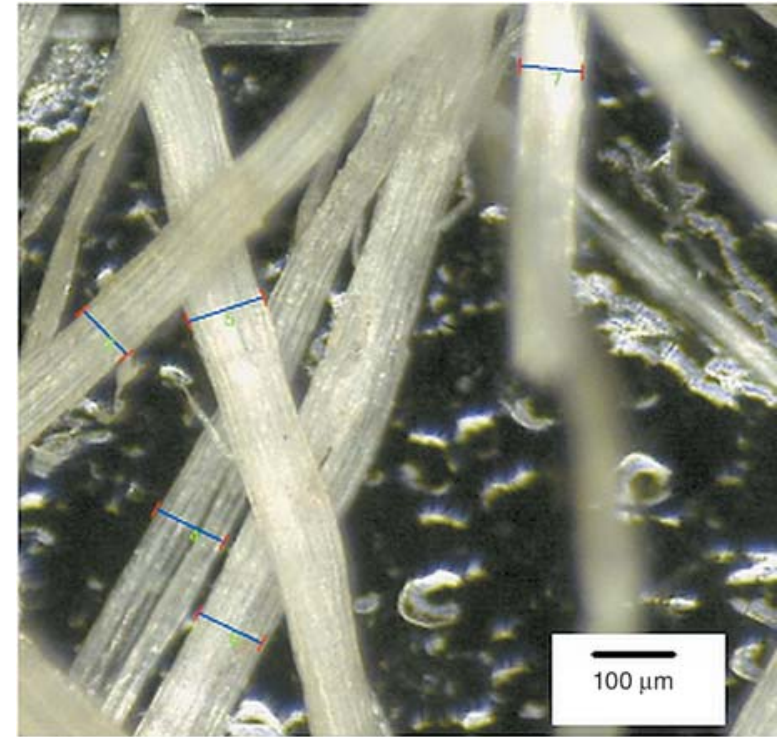

a)

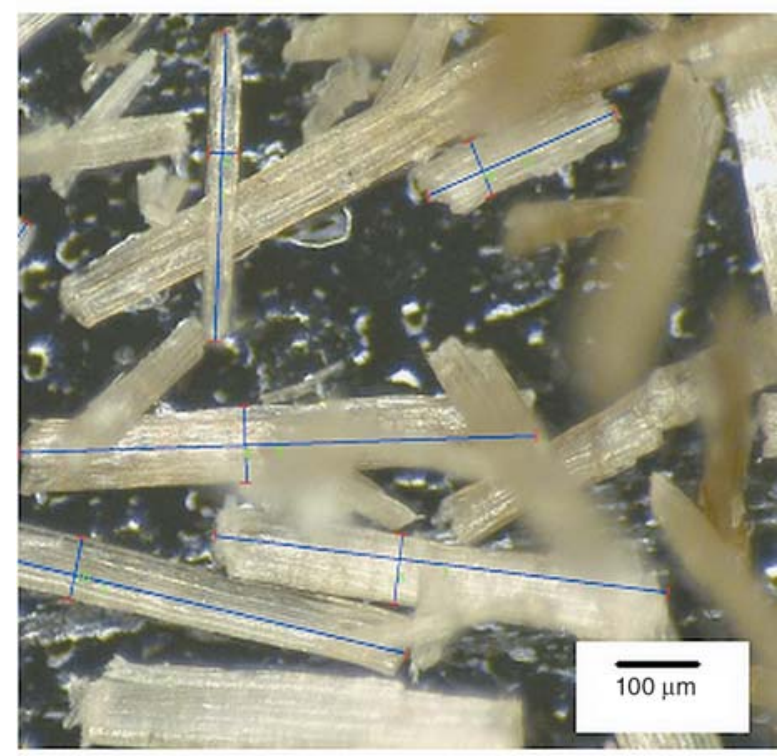

b)

Figure 4. KF fiber diameter before kneading (a) and after kneading (b)

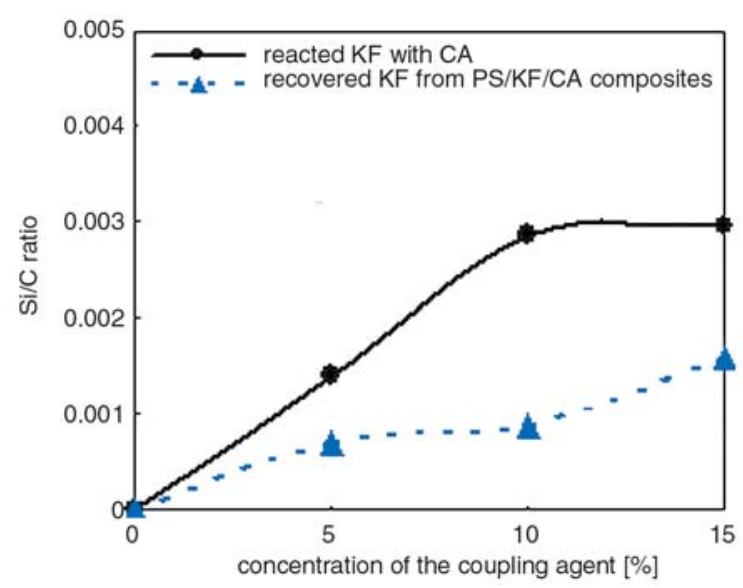

Figure 5. The $\mathrm{Si} / \mathrm{C}$ ratio of the recovered $\mathrm{KF}$ from the composites and the reacted KF analyzed by SEM-EDX 
der Waals interaction with the PS matrix due to their similar chemical structures. Although van der Waals interaction is not as strong as other primary bonds, it could still improve the adhesion properties between hydrophilic KF and the hydrophobic PS matrix.

\subsection{Morphology of the KF}

Scanning electron microscopy provides an excellent technique for examination of surface morphology of fibers and fracture surfaces of the fiber

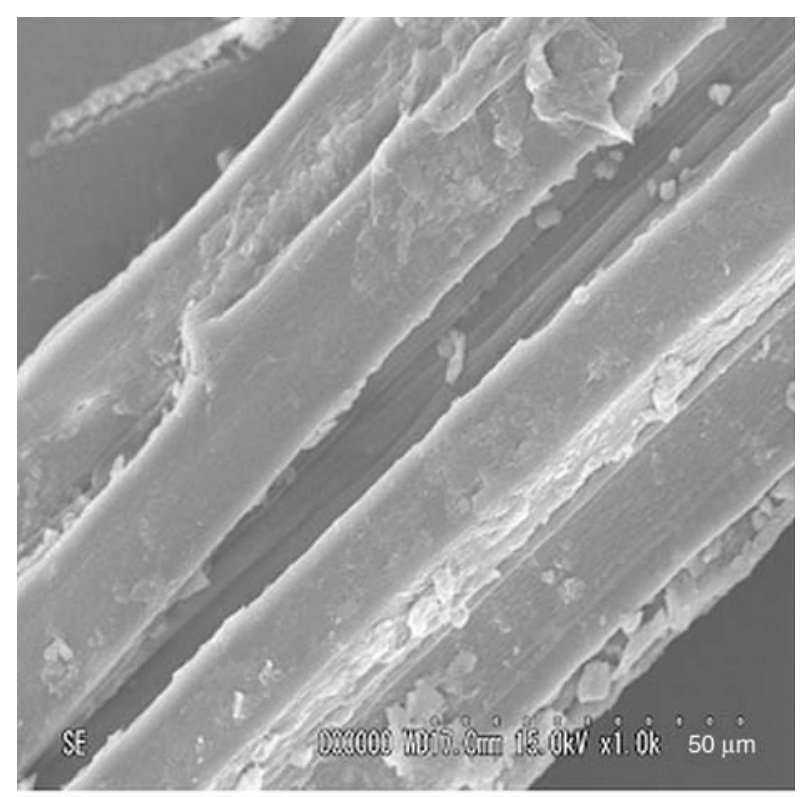

a)

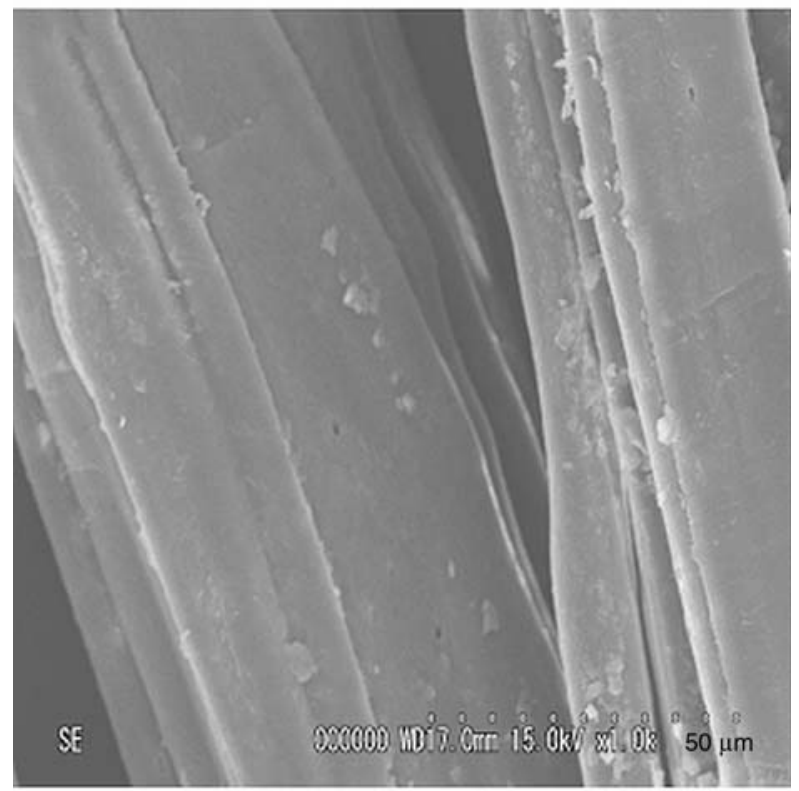

b)

Figure 6. SEM micrographs of longitudinal views of (a) unwashed KF and (b) THF washed KF composites. Examinations were carried out on the unwashed fiber and THF washed fiber.

The SEM micrograph of the longitudinal surface of unwashed KF bundles in Figure 6a shows the presence of wax, oil and surface impurities. The existence of these materials will decrease the mechanical properties of the fiber composites. Figure $6 \mathrm{~b}$ shows the surface of the KF bundles washed with THF. It seems that the impurity has been removed without roughing the surface of fiber bundles.

\subsection{Mechanical properties of composites}

Dynamic mechanical thermal analysis was carried out on the different types of composites prepared. Storage modulus values of the PS, PS/KF/CA composites are compared in Figure 7.

The storage modulus is useful in assessing the molecular basis of the mechanical properties of a material because it is very sensitive to structural changes, such as fiber-matrix interfacial bonding. Figure 7 shows the storage modulus of neat PS, $\mathrm{PS} / \mathrm{KF} / \mathrm{CA}$ composites with $50 \mathrm{wt} \%$ fiber treated in different concentration of CA solution. The storage modulus improved significantly with the addition of KF to PS over the whole range of the testing temperatures as a result of the reinforcement imparted by the fiber. A much enhanced storage modulus of the composites are obtained for the $\mathrm{PS} / \mathrm{KF} / \mathrm{CA}$ composites compared with the untreated $\mathrm{C} 0$ composite which suggests that the adhesion between PS and un-treated KF is very poor as observed in other Polyolefin/KF composites [27] and the surface modification of $\mathrm{KF}$ with $\mathrm{CA}$

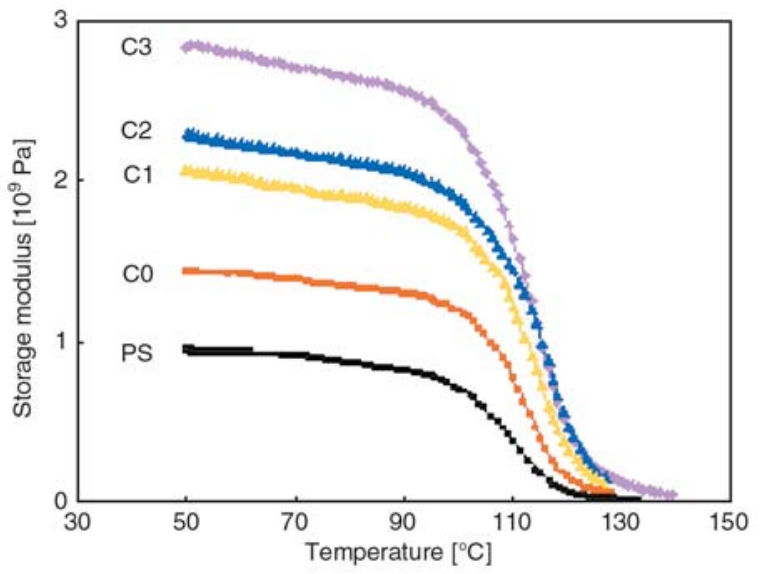

Figure 7. Variation of storage modulus of non-reacted and reacted KF/PS composites as a function of temperature 


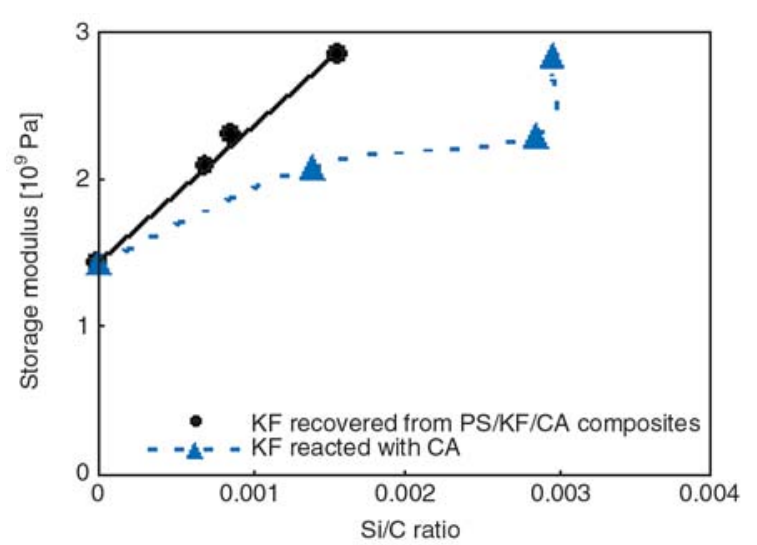

Figure 8. Variation of storage modulus of the PS/KF/CA composites at $50{ }^{\circ} \mathrm{C}$ as a function of $\mathrm{Si} / \mathrm{C}$ ratio

improves the adhesion strength. With the increasing of the $\mathrm{CA}$ content on $\mathrm{KF}$, the storage modulus becomes larger.

Figure 8 shows the variation of storage modulus of the PS/KF/CA composites at $50^{\circ} \mathrm{C}$ as a function of $\mathrm{Si} / \mathrm{C}$ ratio observed on KF. The storage modulus of the composites increases with the $\mathrm{Si} / \mathrm{C}$ ratio, which strongly proved that the modification of fiber with CA contributes to the improvement of interfacial adhesion of KF and PS matrix. It should be noted here that the storage modulus is in proportion to the $\mathrm{Si} / \mathrm{C}$ of recovered KF but not with the reacted KF. As mentioned previously, a part of CA attached to $\mathrm{KF}$ by solution reaction is removed during molding. The observed $\mathrm{Si} / \mathrm{C}$ ratio seems to represent the amount of CA found on KF accurately and to be in accordance with the interaction strength between $\mathrm{KF}$ and PS matrix. According to the modified Kerner equation for the composite with randomly dispersed fibrous filler [28] and using the measured values, the Young's modulus of the present composite $(E)$ is estimated from Equation (2):

$\frac{E}{E_{P S}}=\frac{1+A \cdot B \cdot \phi_{K F}}{1-B \cdot \psi \cdot \phi_{K F}}$

where $E$ is the estimated modulus of the composites, $E_{P S}$ is the modulus of the PS, $\phi_{K F}$ is the volume fraction of KF. $B$ and $\psi$ are given Equations (3) and (4):

$$
B=\frac{\frac{E_{K F}}{E_{P S}}-1}{\frac{E_{K F}}{E_{P S}}+A}
$$

$$
\psi \approx 1+\left(\frac{1-\phi_{m}}{\phi_{m}^{2}}\right) \cdot \phi_{K F}
$$

where $E_{K F}$ is the modulus of the KF. $\phi_{m}$ is the maximum volume of the KF. With the random filling of the $\mathrm{KF}$, the $\phi_{m}$ is 0.8 .

With the random filling of the $\mathrm{KF}, \mathrm{A}$ is determined by the ratio of KF length $(L)$ and diameter $(D)$. In the present study, the $L / D$ is 4 leading to $A$ is 2.08 [29].

Young's modulus of KF has been estimated as $38 \mathrm{GPa}$ [30] and KF density was measured as $1.3824 \mathrm{~g} / \mathrm{cm}^{3}$, respectively.

The value observed for $\mathrm{C} 3$ sample in this present study, $2.86 \mathrm{GPa}$, is about $84 \%$ of the theoretically predicted value, c.a. $3.39 \mathrm{GPa}$, which indicates $\mathrm{CA}$ with graft yield of $5.8 \%$ functioned successfully in the PS/KF composites.

The value of the $\tan \delta$, the ratio of viscous to elastic properties, of $\mathrm{PS} / \mathrm{KF} / \mathrm{CA}$ composites with $50 \mathrm{wt} \%$ fiber are shown in Figure 9 as a function of temperature. It is obvious that the composites are elastic material with $\tan \delta$ close to zero at low temperature below $90^{\circ} \mathrm{C}$. Beyond $90^{\circ} \mathrm{C}$, they become viscous owing to their PS component but there is not a big difference in height of $\tan \delta$ peak among the untreated-fiber composite $\mathrm{C} 0$ and reacted-fiber composites C1-C3 indicating the same order of damping capabilities. The slight reduction in $\tan \delta$ for the reacted-fiber composites means that the mobility of PS molecules is restricted due to the stronger interfacial interaction between KF and PS than that for untreated-fiber composite. Furthermore, the decrease in mechanical loss factor is also denotes an improvement of fatigue property.

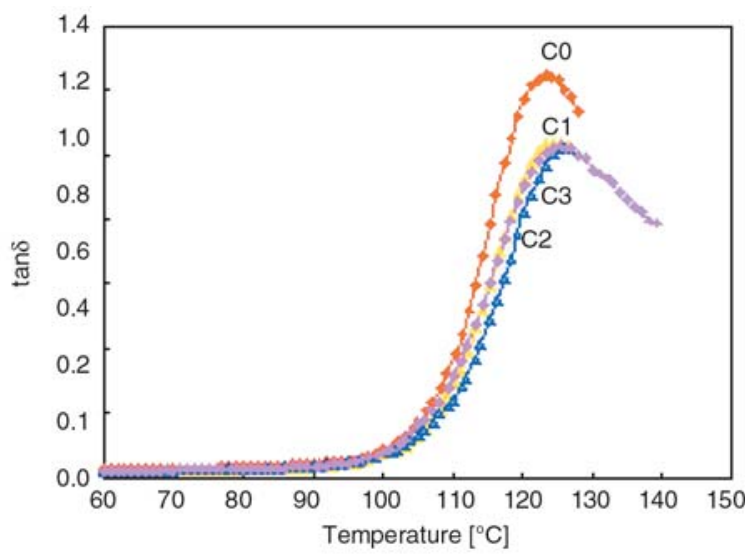

Figure 9. Variation of $\tan \delta$ of untreated and reacted-fiber $\mathrm{PS} / \mathrm{KF} / \mathrm{CA}$ composites with fiber as a function of temperature 


\section{Conclusions}

In this paper, the surface of the KF was modified using a synthesized polymeric coupling agent to promote adhesion with a PS matrix, and the dynamic mechanical thermal analysis of the modified KF on the PS composites was investigated. CA treatment of the KF increased the fiber-matrix interaction through an ester-exchange reaction between alkoxysilane and hydroxyl groups of kenaf cellulose. DMA showed that modified fiber composites have higher $E^{\prime}$ and lower tan $\delta$ indicating a greater interfacial bond strength and adhesion between the matrix resin and the fiber. After kneading, the KF kept the same diameter, but shortened the fiber length and decreased the $\mathrm{Si} / \mathrm{C}$ ratio. The storage modulus increased steadily with the increase of the Si/C ratio on KF surface after kneading. A good consistency between the high Si concentrations on KF with the high mechanical properties of the composites was proved.

\section{Acknowledgements}

This study was supported by TOHO INDUSTRIAL CO.,LTD as a part of the project 'Development of Creative Technology Seeds - Contract Development' promoted by Japan Science and Technology Agency.

\section{References}

[1] Hong C. K., Hwang I., Kim N., Park D. H., Hwang C. B. S., Nah C.: Mechanical properties of silanized jutepolypropylene composites. Journal of Industrial and Engineering Chemistry, 14, 71-76 (2008). DOI: 10.1016/j.jiec.2007.07.002

[2] O'Donnell A., Dwieb M. A., Wool R. P.: Natural fiber composites with plant oil-based resin. Composites Science and Technology, 64, 1135-1145 (2004). DOI: 10.1016/j.compscitech.2003.09.024

[3] Dweib M. A., Hu B., O’Donnell A., Shenton H. W., Wool R. P.: All nature composites sandwich beams for structural applications. Composite Structures, 63, 147-157 (2004). DOI: $\underline{10.1016 / \mathrm{S} 0263-8223(03) 00143-0}$

[4] Munikenche Gowda T., Naidu A. C. B., Rajput Chhaya: Some mechanical properties of untreated jute fabric-reinforced polyester composites. Composites Part A: Applied Science and Manufacturing, 30, 277284 (1999).

DOI: $\underline{10.1016 / S 1359-835 X(98) 00157-2}$
[5] Nishino T., Hirao K., Kotera M.: X-ray diffraction studies on stress transfer of kenaf reinforced poly (Llactic acid) composites. Composites Part A: Applied Science and Manufacturing, 37, 2269-2273 (2006). DOI: 10.1016/j.compositesa.2006.01.026

[6] Shibata S., Cao Y., Fukumoto I.: Lightweight laminate composites made from kenaf and polypropylene fibres. Polymer Testing, 25, 142-148 (2006). DOI: $\underline{10.1016 / j . p o l y m e r t e s t i n g .2005 .11 .007}$

[7] Amaducci S., Amaducci M. T., Benati R., Venturi G.: Crop yield and quality parameters of four annual fiber crops in north of Italy. Industrial Crops and Products, 11, 179-186 (2000). DOI: 10.1016/S0926-6690(99)00063-1

[8] Nishino T., Hirao K., Kotera M., Nakamae K., Inagaki H.: Kenaf reinforced biodegradable composite. Composites Science and Technology, 63, 1281-1286 (2003).

DOI: $10.1016 / \mathrm{S} 0266-3538(03) 00099-X$

[9] Ray D., Sarkar B., Das S., Rana A.: Dynamic mechanical and thermal analysis of vinylester-resin-matrix composites reinforced with untreated and alkalitreated jute fibres. Composites Science and Technology, 62, 911-917 (2002). DOI: $\underline{10.1016 / \mathrm{S} 0266-3538(02) 00005-2}$

[10] Sydenstricker T. H. D., Sandro M., Sandro C. A.: Pullout and other evaluations in sisal-reinforced polyester biocomposites. Polymer Testing, 22, 375-380 (2003). DOI: $\underline{10.1016 / \mathrm{S} 0142-9418(02) 00116-2}$

[11] Eichhorn S. J., Baillie C. A., Zafeiropoulos N., Mwaikambo L. Y., Ansell M. P., Dufresne A., Entwistle K. M., Herrera-Franco P. J., Escamilla G. C., Groom L., Hughes M., Hill C., Rials T. G., Wild P. M.: Review current international research into cellulosic fibres and composites. Journal of Materials Science, 36, 2107-2131 (2001). DOI: $10.1023 / \mathrm{A}: 1017512029696$

[12] Sgriccia N., Hawley M. C.: Thermal, morphological, and electrical characterization of microwave processed natural fiber composites. Composites Science and Technology, 67, 1986-1991 (2007). DOI: 10.1016/j.compscitech.2006.07.031

[13] Wambua P., Ivens J., Verpoest I.: Natural fibres: Can they replace glass in fibre reinforced plastics? Composites Science and Technology, 63, 1259-1264 (2003).

DOI: $\underline{10.1016 / \mathrm{S} 0266-3538(03) 00096-4}$

[14] Toiz G., Denes F., Young R.: Lignin-polypropylene composites. Part 1: Composites from unmodified lignin and polypropylene. Polymer Composites, 23, 806-813 (2002).

DOI: $\underline{10.1002 / p c .10478}$

[15] Fung K. L., Xing X. S., Li R. K. Y., Tjong S. C., Mai Y-W.: An investigation on the processing of sisal fibre reinforced polypropylene composites. Composites Science and Technology, 63, 1255-1258 (2003). DOI: $10.1016 / \mathrm{S} 0266-3538(03) 00095-2$ 
[16] Rezaur Rahman Md., Monimul Huque Md., Nazrul Islam Md., Mahbub Hasan: Mechanical properties of polypropylene composites reinforced with chemically treated abaca. Composites Part A: Applied Science and Manufacturing, 40, 511-517 (2009)

DOI: 10.1016/j.compositesa.2009.01.013

[17] Riccieri J., Vazguez A., de Carvalho L. H.: Interfacial properties and initial step of the water sorption in unidirectional unsaturated polyester/vegetable fibre composites. Polymer Composites, 20, 29-37 (1999). DOI: $\underline{10.1002 / \mathrm{pc} .10332}$

[18] Gauthier R., Joly C., Coupas A., Gaultier H., Escoubes M.: Interfaces in polyolefin/cellulosic fibre composites: Chemical coupling, morphology, correlation with adhesion and aging in moisture. Polymer Composites, 19, 287-300 (1998).

DOI: $10.1002 / \mathrm{pc} .10102$

[19] Gassan J., Bledzki A. K.: Effect of cyclic moisture absorption desorption on the mechanical properties of silanized jute-epoxy composites. Polymer Composites, 20, 604-611 (1999).

DOI: $10.1002 /$ pc. 10383

[20] Dash B. N., Rana A. K., Mishra H. K., Nayak S. K., Mishra S. C., Tripathy S. S.: Novel, low-cost jutepolyester composites. Part 1: Processing, mechanical properties, and SEM analysis. Polymer Composites, 20, 62-71 (1999).

DOI: $\underline{10.1002 / p c .10335}$

[21] Feng D., Caulfield D. F., Sanadi A. R.: Effect of compatibilizer on the structure-property relationships of kenaf-fiber/polypropylene composites. Polymer Composites, 22, 506-517 (2001).

DOI: $10.1002 /$ pc. 10555

[22] Keener T. J., Stuart R. K., Brown T. K.: Maleated coupling agents for natural fibre composites. Composites Part A: Applied Science and Manufacturing, 35, 357367 (2004).

DOI: 10.1016/j.compositesa.2003.09.014
[23] Aziz S. H., Ansell P. M., Clarke S. J., Panteny S. R.: Modified polyester resins for natural fibre composites. Composites Science and Technology, 65, 525-535 (2005).

DOI: 10.1016/j.compscitech.2004.08.005

[24] Shibata S., Cao Y., Fukumoto I.: Press forming of short natural fiber-reinforced biodegradable resin: Effects of fiber volume and length on flexural properties. Polymer Testing, 24, 1005-1011 (2005). DOI: 10.1016/j.polymertesting.2005.07.012

[25] Arbelaiz A., Fernández B., Ramos J. A., Retegi A., Llano-Ponte R., Mondragon I.: Mechanical properties of short flax fibre bundle/polypropylene composites: Influence of matrix/fibre modification, fibre content, water uptake and recycling. Composites Science and Technology, 65, 1582-1592 (2005). DOI: $10.1016 /$ j.compscitech.2005.01.008

[26] Xu Y., Kawata S., Hosoi K., Kawai T., Kubota H., Kuroda S.: Preparation of polymeric coupling agent by means of photo-graft polymerization onto PS microspheres. Journal of Applied Polymer Science, in press (2009).

[27] Sanadi A. R., Caulfield D. F., Jacobson R. E., Rowell R. M.: Renewable agricultural fibers as reinforcing fillers in plastics: Mechanical properties of kenaf fiber-polypropylene composites. Industrial and Engineering Chemistry Research, 34, 1889-1896 (1995). DOI: $\underline{10.1021 / \mathrm{ie} 00044 \mathrm{a} 041}$

[28] Lewis T. B., Nielsen L. E.: Dynamic mechanical properties of particulate-filled composites. Journal of Applied Polymer Science, 14, 1449-1471 (1970). DOI: 10.1002/app.1970.070140604

[29] Nielsen L. E.: Mechanical properties of polymers and composites. Marcel Dekker, New York (1976).

[30] Ochi S.: Mechanical properties of kenaf fibers and kenaf/PLA composites. Mechanics of Materials, 40, 446-452 (2008). DOI: 10.1016/j.mechmat.2007.10.006 